\title{
Exercise Fails to Improve Neurocognition in Depressed Middle- Aged and Older Adults
}

\author{
Benson M. Hoffman ${ }^{1}$, James A. Blumenthal ${ }^{1}$, Michael A. Babyak ${ }^{1}$, Patrick J. Smith ${ }^{1}$, Sharon \\ D. Rogers ${ }^{2}$, P. Murali Doraiswamy ${ }^{1}$, and Andrew Sherwood ${ }^{1}$ \\ ${ }^{1}$ Department of Psychiatry and Behavioral Sciences, Duke University, Durham, NC \\ ${ }^{2}$ Department of Health Education and Promotion, East Carolina University, Greenville, NC
}

\section{Abstract}

Purpose-Although cross-sectional studies have demonstrated an association between higher levels of aerobic fitness and improved neurocognitive function, there have been relatively few interventional studies investigating this relationship, and results have been inconsistent. We assessed the effects of aerobic exercise on neurocognitive function in a randomized controlled trial of patients with major depressive disorder (MDD).

Methods-Two-hundred and two sedentary men $(n=49)$ and women $(n=153)$, aged $40 \mathrm{yr}$ and over and who met diagnostic criteria for MDD, were randomly assigned to the following: a) supervised exercise, b) home-based exercise, c) sertraline, or d) placebo pill. Before and after 4 months of treatment, participants completed measures of: Executive Function (Trail Making Test BA difference score, Stroop Color/Word, Ruff $2 \& 7$ Test, Digit Symbol), Verbal Memory (Logical Memory, Verbal Paired Associates), and Verbal Fluency/Working Memory (Animal Naming, Controlled Oral Word Association Test, Digit Span). Multivariate analyses of covariance were performed to test the effects of treatment on posttreatment neuropsychological test scores, with baseline neuropsychological test scores, age, education, and change in depression scores entered as covariates.

Results-The performance of exercise participants was no better than participants receiving placebo across all neuropsychological tests. Exercise participants performed better than participants receiving sertraline on tests of executive function but not on tests of verbal memory or verbal fluency/ working memory.

Conclusions-We found little evidence to support the benefits of an aerobic exercise intervention on neurocognitive performance in patients with MDD.

\section{Keywords}

FITNESS; DEPRESSION; COGNITIVE FUNCTION; EXECUTIVE FUNCTION

\begin{abstract}
In addition to the well-documented medical benefits of physical exercise (62), the value of exercise for improving mental health also has been increasingly recognized (59). Published reviews have concluded that exercise interventions are associated with improved psychological well-being, life satisfaction, and self-efficacy (44) as well as reduced symptoms of anxiety (51) and depression (5). Among clinically depressed populations, strength training (58) and aerobic exercise (20) have been associated with reduced depressive symptoms and remission
\end{abstract}


of major depressive disorder (MDD). In previous reports from our laboratory, exercise was shown to be comparable to antidepressant medication in reducing depressive symptoms among clinically depressed adults (10), and exercise was shown to be associated with lower risk for relapse in remitted patients (4). Recently, we conducted a randomized controlled trial and reported that MDD remission rates for exercisers were comparable to participants taking antidepressant medication and were better than placebo pill control (9).

Studies evaluating the effects of exercise on neurocognitive function have been more equivocal. Cross-sectional studies have generally shown that aerobically fit individuals have higher scores on measures of perceptual speed, reasoning, working memory, and executive function (i.e., the cognitive ability for volition, planning, purposive action, and effective performance (40)) compared with their sedentary counterparts (35). Despite the paucity of randomized controlled trials, a recent meta-analysis of exercise studies on older adults concluded that exercise was associated with improved cognitive performance, particularly on tasks that assess executive functions (15). However, results from randomized controlled trials have been inconsistent; some studies have shown improvements in cognition $(21,39,54)$, whereas others have not $(11,14,30,34)$.

Aerobic fitness is thought to impact neurocognition by altering brain structure and function (17). In animal studies, aerobic exercise has been associated with increased cortical capillary supplies, formation of new synaptic connections, and growth of new neurons, particularly in the hippocampus (38). Mechanisms may include neurochemicals such as brain-derived neurotrophin factor and insulin-like growth factor $(17,38)$. A recent study provided preliminary evidence for an exercise-related growth in cerebral blood volume of the human hippocampus, suggesting that exercise may benefit humans and mice through similar mechanisms (49). However, a recent meta-regression of cross-sectional and longitudinal exercise studies in humans found no evidence for an association between improved fitness and improved neurocognitive function in humans (24).

As part of a larger randomized clinical trial (9), we examined the effects of aerobic exercise relative to placebo pill and antidepressant medication on neurocognitive function in a sample of sedentary, clinically depressed, middle-aged and older adults. We hypothesized that exercise participants would have greater improvement in neuropsychological test performance compared with participants receiving either antidepressant medication or placebo pill. We based our hypothesis that exercise, but not medication, would be associated with improved neurocognitive performance on the belief that such improvement would not be mediated by changes in depressive symptoms but rather by exercise-induced biologic changes (as described above). In a previous study conducted by our research team, clinically depressed adults who underwent aerobic exercise training exhibited greater improvements on the Stroop Interference Task compared with participants receiving sertraline (37). Thus, we believed that exercise training would result in improvements in neurocognitive test performance on tasks that tap more executive functions.

\section{METHOD}

Participants

The details of participant recruitment and experimental design are presented elsewhere (9). Briefly, participants who gave written informed consent for participation in this IRB-approved study, who had scores of 12 or greater on the Beck Depression Inventory-2 (BDI) (7), and who met diagnostic criteria for MDD using the Structured Clinical Interview for the Diagnostic and Statistical Manual of Mental Disorders, Fourth Edition (2) and the Hamilton Depression Rating Scale (HAM-D) (29) completed an assessment battery that included measures of 
cardiopulmonary fitness and neurocognitive function. Bipolar disorder, psychotic depression, and significant cognitive impairment were excluded.

\section{Assessment of neurocognitive function}

A neuropsychological test battery was individually administered to all participants by a trained research technician who was blind to treatment group assignment. The battery was composed of tests selected for their use in prior studies, availability of normative data, and measurement of different cognitive domains, especially executive function. Selected tests included the following:

Animal Naming (40). Participants name as many animals as possible in $60 \mathrm{~s}$. The animal naming score is the total number of animals named. This test primarily assesses verbal fluency but is also reflective of executive function and memory.

Controlled Oral Word Association Test (COWAT) (40). Participants generate as many words as possible that begin with a specific letter in $60 \mathrm{~s}$. This task is repeated three times with three different letters. This test measures verbal fluency and also draws on executive function and memory. For the current study, the three letters alternated between "CFL" and "PRW" to minimize practice effects.

Digit Symbol Subtest from the Wechsler Adult Intelligence Scale-Revised (WAIS-R) (63). Participants draw symbols that match one of 10 digits copied from a key. One point is awarded for each correct symbol drawn in $90 \mathrm{~s}$, with a maximum score of 90 . This test measures psychomotor speed and working memory.

Digit Span Subtest from the WAIS-R (64). Digit span consists of two subtests. Digits forward requires participants to repeat increasingly long strings of verbally presented numbers. This is a test of short-term memory storage capacity (40). Digits backward requires participants to repeat digits in reverse order, an added complexity that places demand on working memory.

Ruff $2 \& 7$ Test (55). Participants are provided with 20 sets of characters. In 10 sets, the numbers "2" and "7" are embedded within other numbers. In 10 sets, the numbers "2" and "7" are embedded within a series of capital letters. Each trial lasts $15 \mathrm{~s}$. Total score equals the number of targets hit minus errors of omission and commission. This test requires rapid visual discrimination and intact executive function.

Stroop Color and Word Test-Interference List (61). The Stroop test consists of three lists: a word list, a color list, and an interference list. The word list requires participants to read a list of color words as quickly as possible, with the score being the total number of words read aloud in $45 \mathrm{~s}$. The color list requires participants to name aloud the color of each of a series of colored bars in $45 \mathrm{~s}$. The interference list consists of a series of color words, but the words themselves are colored in a different color ink than the color to which they refer. Participants are required to name the color of the word but not the word itself. This is considered a test of executive function.

Trail Making Test (TMT) (40). This test was originally part of the Army Individual Test Battery. In Part A, participants are required to connect a series of 25 numbered dots. In Part $B$, participants are required to connect a series of alternating numbered and lettered dots. The difference between time to complete Part B and Part A (TMT B-A) is considered a measure of executive function.

Verbal Paired Associates Subtest from the Wechsler Memory Scale (WMS) (64).

Participants are orally presented with eight word pairs. The four word pairs from the "easy" list are associated (e.g., baby-cries), whereas the four pairs from the "hard" list are not 
(e.g., school-grocery). Participants are then presented with individual words from each pair and are asked to recall the paired word.

Logical Memory Subtest from the WMS (64). Participants are required to repeat a story read aloud to them. Immediate recall was scored using a verbatim scoring procedure. This test measures short-term semantic memory.

\section{Assessment of physical fitness}

Graded treadmill exercise testing was conducted before and after treatment. Patients exercised to exhaustion or standard end points (e.g., >2 mm ST-segment depression, abnormal blood pressure response, etc.) under continuous electrocardiographic monitoring using the DukeWake Forest protocol in which workloads are increased at a rate of $1 \mathrm{METImin}^{-1}(13)$. Expired air was collected by mouthpiece for quantification of minute ventilation, oxygen consumption, and carbon dioxide production with a Beckman Metabolic Cart (Model 040-301, Fullerton, CA). Samples were collected at 20-s intervals, and peak values were determined from an average obtained during the last $60 \mathrm{~s}$.

\section{Interventions}

Participants were randomized to one of four treatment groups:

1. Supervised Aerobic Exercise. Participants exercised three times per week for 16 consecutive weeks. They were assigned individual training ranges equivalent to 70 $85 \%$ of their HR reserves, which was calculated from the maximum HR achieved during the initial treadmill test. HR and RPE were recorded three times during each exercise session by a trained exercise physiologist.

2. Home-Based Aerobic Exercise. Participants received an initial exercise training session with an exercise physiologist. During this session, participants received an individualized exercise prescription, with target ranges equivalent to $70-85 \%$ of their HR reserves, as well as guidance regarding how to implement their exercise program. Participants received two follow-up sessions with an exercise physiologist for the purposes of adherence promotion and performance correction. Participants monitored and recorded their HR and RPE during exercise sessions on weekly exercise logs.

3. Sertraline or 4. Placebo Pill. Participants received sertraline (Zoloft), a selective serotonin-reuptake inhibitor, or placebo pill. Both participants and study staff were blind as to whether participants received medication or placebo. Participants met with a staff psychiatrist at study onset and weeks 2, 4, 8, 12, and 16. Treatment was initiated at $50 \mathrm{mg}$ and titrated until a well-tolerated therapeutic dosage was achieved, up to 200 $\mathrm{mg}$ (four pills). Participants in the pill conditions were instructed to refrain from any regular exercise until the conclusion of the study.

\section{Data analysis}

Principal axis factor analysis with an oblique Promax rotation was used to group the neuropsychological tests into distinct neurocognitive domains for analyses. The factors were labeled Executive Function (TMT B-A, Stroop Color-Word, Ruff $2 \& 7$ Test, Digit Symbol), Verbal Memory (Logical memory, verbal paired associates—easy, verbal paired Associateshard), and Verbal Fluency/Working Memory (Animal Naming, COWAT, Digits Forward, Digits Backward). Factor loadings can be found in Table 1.

Our primary analyses compared neurocognitive outcomes by treatment group. Three separate MANCOVA were performed, one for each neurocognitive domain, entering baseline neuropsychological test scores, education, age, and change in depression (baseline HAM-D minus posttreatment HAM-D) as covariates. Treatment condition served as the grouping 
variable. Because we had no a priori reason to believe that home-based and supervised exercise would differentially impact neurocognitive function, the supervised and the home-based exercise conditions were combined into a single exercise group. Unlike placebo, sertraline has direct central nervous system effects that affect neurocognitive function, so the pill groups were not combined. Significant univariate treatment effects were examined with planned contrasts (i.e., exercise vs placebo; exercise vs sertraline). Our primary data analyses used the intent-to-treat principle; the last observation was carried forward for 23 participants who were missing posttreatment data.

To further examine the impact of exercise, we also conducted two sets of exploratory analyses using restricted data sets. We performed a treatment completers analysis, in which the three MANCOVA were repeated using a data set restricted to those participants who did not formally withdraw from the study or informally discontinue their treatment regimen and who completed posttreatment testing. We also performed an optimal exercisers analysis, in which we compared those exercise participants in the top tertile of improvement in aerobic fitness (i.e., the largest percent improvement in peak $\mathrm{VO}_{2}$ after exercise) with those patients receiving placebo.

All analyses were performed with SAS v 9.1 (56).

\section{RESULTS}

\section{Sample Characteristics}

The sample consisted of 202 participants (age mean $=51.7 \mathrm{yr} ; \mathrm{SD}=7.6$ ), the majority of whom were female (76\%) and Caucasian (68\%). The sample had an average of $15.8 \mathrm{yr}$ of education $(\mathrm{SD}=2.7)$. After 4 months of treatment, participants in all treatment groups experienced decreased symptoms of depression as measured by the HAM-D and the BDI, and participants receiving active treatments tended to have higher remission rates than placebo controls. Results of the primary analyses concerning the effects of exercise on depression are reported elsewhere (9). Sample characteristics by group can be found in Table 2.

\section{Treatment Adherence}

One hundred and four participants were randomized to an exercise condition, whereas 49 were randomized to the sertraline condition and 49 were randomized to the placebo condition.

Among exercise participants, 13 (13\%) formally withdrew from the study, and another 8 (8\%) remained in the study but discontinued exercise treatment. Treatment completers attended an average of 38.8 exercise sessions.

Adherence to medication treatment was evaluated by pill count. The mean maximum dose of medication prescribed over the course of the study was similar for the sertraline and the placebo groups (mean $=131 \mathrm{mg}$ for the sertraline group and $133 \mathrm{mg}$ for the placebo group). There were 7 dropouts (14\%) in the sertraline condition and 14 dropouts (29\%) in the placebo condition.

\section{Effects of Treatment on Aerobic Capacity}

Participants in the exercise groups displayed higher levels of aerobic capacity $\left(\right.$ peak $\mathrm{VO}_{2}$ ) and greater treadmill times compared with participants who received pills ( $P$ 's $<0.0001)$. Differences in aerobic capacity and treadmill endurance also were noted between exercise treatment conditions, with supervised exercise participants achieving higher levels of aerobic capacity and longer treadmill times than those participants who exercised at home ( $P$ 's < 0.0001 ). The participants in home-based exercise showed an improvement of $3.5 \%$ on peak $\mathrm{VO}_{2}$ and $7.5 \%$ on treadmill time; the supervised exercise improved by $8 \%$ on peak $\mathrm{VO}_{2}$ and $19 \%$ on treadmill time; the sertraline group showed a $1 \%$ decrease in peak $\mathrm{VO}_{2}$ and $4 \%$ 
improvement in treadmill time; and the placebo group declined by $4 \%$ on peak $\mathrm{VO}_{2}$ and $2 \%$ on treadmill time (see Table 3).

\section{Effects of Treatment on Neurocognitive Function}

Pretreatment and posttreatment neurocognitive test scores by treatment group are presented in Table 4. Mean neuropsychological test values for each treatment group are well within normal limits (i.e., + $1 \mathrm{SD}$ ), both before and after treatment, as compared with demographically adjusted normative data $(19,26,32,55,63,64)$. These data suggest that the present sample is reasonably representative of the general population.

The MANCOVA comparing measures of Executive Function revealed a significant treatment main effect $(P=0.014)$. Examination of the univariate ANCOVA revealed significant treatment main effects for TMT B-A $(P=0.046)$ and Ruff Total Score $(P=0.005)$, and a marginally significant treatment main effect for Digit Symbol $(P=0.089)$. Examination of the simple contrasts revealed that exercisers had better scores compared with participants in the sertraline group on TMT B-A $(P=0.02)$, Ruff total score $(P=0.002)$, and digit symbol $(P=$ $0.03)$. Simple contrasts between exercisers and placebo group participants were not significant.

Neither the MANCOVA comparing memory scores by treatment group nor the MANCOVA comparing verbal fluency/working memory scores by treatment group were significant.

Treatment completers analysis-The three MANCOVA were repeated using only available data from treatment completers (exercisers, $n=82$; sertraline, $n=41$; and placebo, $n=35)$. The MANCOVA comparing executive function scores by treatment group revealed a marginally significant treatment main effect $(P=0.078)$. Examination of the univariate ANCOVA revealed a significant treatment main effect for Ruff total score $(P=0.01)$. Simple contrasts revealed that exercisers had higher Ruff total scores compared with sertraline group participants $(P=0.005)$. The simple contrast between exercisers and placebo group participants was not significant. None of the other univariate tests comparing executive function scores by treatment group were significant.

Neither the MANCOVA comparing memory scores by treatment group nor the MANCOVA comparing verbal fluency/working memory scores by treatment group revealed significant treatment main effects.

Optimal exercisers analyses-The three MANCOVA were repeated in which the top tertile of exercisers $\left(n=27\right.$; average change in peak $\left.\mathrm{VO}_{2}=15.60 \%\right)$ were compared with all participants receiving placebo $\left(n=35\right.$; average change in peak $\left.\mathrm{VO}_{2}=-2.94 \%\right)$. The MANCOVA comparing verbal fluency/working memory scores by treatment group revealed a marginally significant treatment main effect $(P=0.06)$. Examination of the univariate ANCOVA revealed a significant treatment main effect for animal naming $(P=0.04)$; exercisers had higher posttreatment animal naming scores compared with placebo group participants. None of the other univariate tests comparing verbal fluency/working memory scores by treatment group were significant.

Neither the MANCOVA comparing memory scores by treatment group nor the MANCOVA comparing executive function scores by treatment group revealed any significant treatment main effects.

Post hoc analysis of age-Our sample was somewhat younger as compared with samples from previous studies of exercise and neurocognitive function. Therefore, we decided to examine the impact of participant age on our primary analyses in a series of post hoc analyses. We observed that the inclusion of age as a covariate did not alter the results of our multivariate 
or univariate analyses. Also, we examined bivariate correlation coefficients between age and change in individual neuropsychological test score (i.e., posttest minus pretest), and none were found to be significant ( $P$ 's $>0.10$ ). Finally, we performed a post hoc analysis in which our primary analyses were repeated using a data set restricted to those participants who were older than $55 \mathrm{yr}(n=38$ exercise, 15 sertraline, and 15 placebo; sample age mean (SD) $=60(5)$; range, 55-81 yr). None of the MANCOVA were significant ( $P$ 's $>0.10)$.

Post hoc analysis of depression-Because our sample was clinically depressed, we decided to examine the impact of participant depression on our primary analyses in a series of post hoc analyses. We observed that the inclusion of change in depression as a covariate did not alter the results of our multivariate or univariate analyses. Similarly, the inclusion of depression severity as a covariate, assessed by either interview (HAM-D) or self-report (BDI), did not alter the results of our multivariate or univariate analyses, regardless of whether depression was measured at baseline or at posttest. Examination of bivariate correlation coefficients revealed that reductions in depressive symptom severity were weakly associated with improved neurocognitive performance and achieved statistical significance on only 2 of 11 tests (Ruff total, $r=0.16, P=0.02$; digit symbol, $r=0.15, P=0.02$ ). Finally, we performed a post hoc analysis in which our primary analyses were repeated using a data set restricted to those participants who were at least moderately depressed (HAM-D >17; $n=38$ exercise, 18 sertraline, and 22 placebo; sample baseline HAM-D mean $(\mathrm{SD})=21.1(2.8))$. None of the MANCOVA were significant $(P$ 's $>0.10)$.

\section{DISCUSSION}

Surprisingly, we found little evidence to support the contention that aerobic exercise training improves neurocognitive function in patients with MDD. We hypothesized that exercise participants would have larger improvements in neuropsychological test performance compared with patients receiving sertraline or placebo pill, especially on tests of executive function. However, exercisers did not perform better on any of the neuropsychological measures compared with placebo controls. Thus, our findings do not provide support for the beneficial effects of exercise training on neurocognitive functioning.

That exercisers had higher executive function scores when compared with the sertraline group, but not when compared with the placebo group, was an unexpected finding. Indeed, whereas both exercisers and placebo controls appeared to improve on some tests of executive function (i.e., Ruff $2 \& 7$ test and digit symbol), which could be attributed to practice effects or greater familiarity with the test procedures, sertraline participants did not appear to improve on these tests. Although sertraline has been associated with improved neurocognitive test performance among depressed adults (18), some studies have reported that sertraline may be associated with very mild neurocognitive decrements (27). In the present study, the higher executive function test scores among participants in the exercise group compared with sertraline group participants appears to be due to the lower performance among patients on sertraline rather than to improvements in neurocognition resulting from exercise. Taken together, data from the present study of patients with MDD do not provide support for an exercise-related improvement in executive function nor in any other neurocognitive domain.

We observed a trend toward enhanced performance for exercise compared with placebo pill in an exploratory analysis of optimal exercisers (i.e., exercisers in the top tertile of improvement in aerobic fitness) on measures of verbal fluency in working memory. However, whereas exercisers had higher scores on one test (i.e., animal naming) within this neurocognitive domain, exercisers did not have higher scores on the other three tests within the same neurocognitive domain (the placebo group actually had slightly higher mean scores) or on neurocognitive tests from other domains. In the absence of a cogent explanation for 
improvement in animal naming, but not in other tests of generative naming or executive function, we conclude that this finding is likely due to chance and is not considered meaningful.

The present findings appear to conflict with the conclusions of a recent meta-analysis (15), which reported that aerobic fitness training interventions improve neurocognitive function of older, sedentary adults by an average of $0.48 \mathrm{SD}$, "regardless of the type of cognitive task, the training method, or participants' characteristics" (p. 128). In that analysis, the reported effect size for neurocognitive tests of executive function ( $0.68 \mathrm{SD}$ for treatment groups vs approximately $0.11 \mathrm{SD}$ for control groups) was especially large. However, closer examination of the 18 studies included in the meta-analysis suggests an inconsistent pattern of results, in which we considered 5 to be positive $(21,31,39,52,54), 6$ equivocal $(6,23,37,43,47,66)$, and 7 negative $(14,22,30,34,41,45,50) .{ }^{1}$ Among the five positive studies, we considered one to have limited generalizability because the clinical population studied had medical conditions that would greatly impact their neuropsychological test performance (i.e., institutionalized psychiatric patients (52)), whereas among the four remaining positive studies, only one used a well-validated set of clinical neuropsychological instruments (21). Two of seven negative studies $(14,22)$ and two of six equivocal studies $(23,37)$ also used well-validated neuropsychological test batteries. Thus, our review of these 18 published exercise intervention studies suggests that there is no consistent evidence supporting a relationship between improved aerobic fitness and cognitive function.

Another recent meta-analysis of 37 studies by Etnier et al. (24) included eight randomized controlled trials that were not included in the Colcombe and Kramer meta-analysis. Examination of these eight studies suggests a similarly inconsistent pattern of findings, in which we considered none to be positive, three to be equivocal $(25,36,48)$, and five to be negative (Russell, E. M., "unpublished manuscript/observations," 1982; 8,12,65,68). ${ }^{2}$ Again, our analysis suggests that there is no consistent evidence in the literature demonstrating a relationship between improved aerobic fitness and cognitive function.

Although the present sample is generally younger than the samples included in the Colcombe and Kramer meta-analysis, it is unlikely that the age of our sample accounted for the discrepancy between our findings and the findings of Colcombe and Kramer. Although older age is generally associated with lower scores on measures of neurocognitive function, especially tests of fluid intelligence or executive functioning (40), there is little evidence that age moderates the relationship between aerobic fitness and neurocognition either in our study or in other published studies. For example, the recent meta-regression by Etnier et al. (24) did not find evidence that age moderates a fitness-neurocognition relationship. Furthermore, the Etnier et al. meta-analysis included four studies with samples that were similar to or younger than those in the present study $(8,12,36,68)$, none of which produced positive findings. Also, with the exception of three studies $(23,47,52)$, the studies included in the Colcombe and Kramer meta-analysis enrolled healthy adults so that the presence of significant cognitive impairment or sufficiently low neuropsychological test scores so as to produce a "floor effect" among these participants was unlikely. Similarly, the present sample of depressed adults had neuropsychological test scores that are well within \pm 1 SD of age, education, and/or gendercorrected normative data. Thus, it is unlikely that age is to account for the discrepancy between our negative findings and Colcombe and Kramer's conclusions (15).

\footnotetext{
1"Positive" studies feature either a majority of positive findings for exercise among a minority of null findings or a pattern of positive and null findings that were predicted a priori. "Negative" studies feature no positive findings for exercise. "Equivocal" studies have a minority of positive findings for exercise among a majority of null findings. We considered (47) to be equivocal due to the absence of a control group. A table detailing these findings can be found online at http://www.duke.edu/web/behavioralmed/hoffman/HoffmanTable5.pdf.

${ }^{2} \mathrm{~A}$ table detailing these findings can be found online at http://www.duke.edu/web/behavioralmed/hoffman/HoffmanTable6.pdf.
} 
There is another recent meta-analysis of exercise and neurocognitive function (33). However, this meta-analysis is exclusively focused on older adults with dementia or significant neurocognitive impairment. Results of exercise interventions for individuals with such severe neurocognitive deficits are unlikely to generalize to outpatient, healthy adults. Therefore, studies from this meta-analysis were not considered relevant to the findings of our present study.

Our failure to find an exercise-induced improvement in neurocognitive functioning in patients with MDD raises questions about the robustness of the relationship of improved aerobic fitness and enhanced neurocognition. Because it is difficult to compare results across studies in which measures of neurocognitive function vary widely between studies, we recommend that researchers adopt a core set of neuropsychological instruments in future studies and examine the impact of exercise on neurocognition in both clinical and healthy populations.

\section{Limitations}

Findings from the present analysis should be interpreted with several limitations in mind. MDD has been associated with suppressed neuropsychological test performance $(28,40)$, and it is possible that MDD obscured the impact of exercise on neurocognitive function in our sample. However, a closer look at our sample suggests that the impact of MDD on neuropsychological test scores likely was limited. Previous studies have shown that the relationship between MDD and neurocognitive function is strongest when depression is severe (42), recurrent (46), accompanied by psychotic features (57), inadequately treated (28), treatment resistant (67), or has a late-life onset (53). However, our post hoc analyses suggested that depression did not have a significant impact on neurocognitive functions in this study.

One possible explanation for weak relationship between depression and neurocognitive function in our sample is that patients with MDD in the present study were not severely depressed. The average participant in this study suffered from depression that was mild to moderate in severity (the average HAM-D score was 17), nonrecurrent ( $58 \%$ had $<2$ prior depressive episodes), early onset (i.e., before age 40), and generally responsive to treatment (e.g., HAM-D scores declined by over $1 \mathrm{SD}$ in each treatment group). In previous studies of adults with uncomplicated, mild-to-moderate depression, the relationship between depression severity and neurocognitive has been weak (1) and has not been consistently observed across studies (3). Although we believe that the impact of MDD on neurocognitive test scores was minimal, we nevertheless cannot rule out the possibility that depression affected our findings and that the lack of exercise-related improvements in neurocognitive function many not generalize to non-depressed patients.

It also is possible that the clinical neuropsychological instruments used in the present study are not sufficiently sensitive to the specific effects of exercise on neurocognitive function. Indeed, three of the five positive studies from the Colcombe and Kramer meta-analysis (31, $39,54)$ and one more recent study (16) observed positive effects for exercise primarily by using computer-based flanker tests, which contrast simple and complex reaction time to assess executive function. Although computer-based flanker tests have been used in research laboratories and validated against brain imaging (16), they typically are not included in standard neuropsychological test batteries (60) and were not used in the present study. It is uncertain whether improvement on a flanker test, particularly in the setting of stable performance on other measures of executive function, affects everyday memory, decision making, or quality of life. We recommend further validation of this finding and future research examining its relevance for daily living.

Another limitation of the present study is the relatively short duration of treatment. Although 4 months is sufficient to improve aerobic capacity and reduce depression $(9,10)$, it may be too 
brief to impact neurocognitive functioning. Indeed, in two of the more noteworthy studies, improved neurocognitive function was achieved in exercise intervention studies lasting 6 months (39) and 3 yr (54). Furthermore, the Colcombe and Kramer meta-analysis (15) concluded that longer intervention periods are associated with increased effect sizes. Longerterm studies of exercise and neurocognitive function may produce greater benefits.

The overall 6\% improvement in aerobic capacity achieved by exercise participants in our study is quite modest and may not have been sufficiently large to impact neurocognitive functioning. However, Kramer et al. found positive effects for exercise on executive functioning with an improvement in aerobic capacity of only $5.1 \%$ (39). Furthermore, a recent meta-analysis found no association between change in aerobic fitness and neurocognitive function in intervention studies (24), and we found little evidence of an exercise benefit even among our optimal exercisers, who achieved $>15 \%$ improvement in aerobic capacity. Although one interventional study showed a dose-response relationship between aerobic exercise amount and changes in depressive symptoms among clinically depressed adults (20), no randomized controlled trial has tested the dose-response relationship between aerobic fitness and cognitive performance.

It is possible that aerobic exercise offers a neurocognitive benefit for people who suffer from cognitive impairment. Indeed, a recent meta-analysis of exercise studies on people with dementia and related cognitive impairment concluded that exercise was associated with improved cognitive performance (33). Because participants in our sample were not cognitively impaired, our null findings may not generalize to a cognitively impaired population.

\section{SUMMARY}

In summary, these findings suggest that exercise does not confer clinically meaningful improvements in neurocognitive function among clinically depressed adults. Exercise offered no clear benefit relative to placebo pill on any of the neuropsychological tests we used in this study. Although exercise was associated with slightly better performance relative to sertraline on a subset of tests, this could be attributed to sertraline-related decrement rather than exerciserelated improvement.

\section{Acknowledgments}

The authors wish to thank Erin Sheets and Chavala Harris for their technical assistance, Marcus Taylor, Jessica Tucker, and Sandra Kennedy for their assistance with exercise testing and training, and all of our study participants.

This study was supported by grant MH 49679 from the National Institutes of Health and grant M01-RR-30 from the General Clinical Research Center Program, National Center for Research Resources, National Institutes of Health. Medication and matched placebo pills were provided by a grant from Pfizer Pharmaceuticals, Inc.

Conflict of interest: Dr Doraiswamy has received grants and honoraria from several pharmaceutical companies. Dr Blumenthal previously received an investigator-initiated research grant from Pfizer/Eisai for an unrelated study.

\section{REFERENCES}

1. Airaksinen E, Larsson M, Lundberg I, Forsell Y. Cognitive functions in depressive disorders: Evidence from a population-based study. Psychol Med 2004;34(1):83-91. [PubMed: 14971629]

2. American Psychiatric Association. Diagnostic and Statistical Manual of Mental Disorders, IV: Text Revision. Vol. 4th ed.. Washington, DC: American Psychiatric Association; 2004. p. 369-428.

3. Austin MP, Mitchell P, Goodwin GM. Cognitive deficits in depression: possible implications for functional neuropathology. Br J Psychiatry 2001;178:200-206. [PubMed: 11230029]

4. Babyak M, Blumenthal JA, Herman S, et al. Exercise treatment for major depression: maintenance of therapeutic benefit at 10 months. Psychosom Med 2000;62(5):633-638. [PubMed: 11020092] 
5. Barbour KA, Blumenthal JA. Exercise training and depression in older adults. Neurobiol Aging 2005 26;:119-123. [PubMed: 16223547]

6. Barry AJ, Steinmetz JR, Page HF, Rodahl K. The effects of physical conditioning on older individuals. II. Motor performance and cognitive function. J Gerontol 1966;21(2):192-199. [PubMed: 5930513]

7. Beck, A.; Steer, R.; Brown, G. BDI-II Manual. San Antonio, TX: Psychological Corporation; 1996. p. 1-38.

8. Blaney J, Sothmann M, Raff H, Hart B, Horn T. Impact of exercise training on plasma adrenocorticotropin response to a well-learned vigilance task. Psychoneuroendocrinology 1990;15(56):453-462. [PubMed: 1966301]

9. Blumenthal JA, Babyak MA, Doraiswamy MP, et al. Exercise and pharmacotherapy in the treatment of major depressive disorder. Psychosom Med 2007;69:587-596. [PubMed: 17846259]

10. Blumenthal JA, Babyak MA, Moore KA, et al. Effects of exercise training on older patients with major depression. Arch Intern Med 1999;159(19):2349-2356. [PubMed: 10547175]

11. Blumenthal JA, Emery CF, Madden DJ, et al. Cardiovascular and behavioral effects of aerobic exercise training in healthy older men and women. J Gerontol 1989;44(5):M147-M157. [PubMed: 2768768]

12. Blumenthal JA, Madden DJ. Effects of aerobic exercise training, age, and physical fitness on memorysearch performance. Psychol Aging 1988;3(3):280-285. [PubMed: 3268270]

13. Blumenthal JA, Rejeski WJ, Walsh-Riddle M, et al. Comparison of high- and low-intensity exercise training early after acute myocardial infarction. Am J Cardiol 1988;61(1):26-30. [PubMed: 3337013]

14. Blumenthal JA, Emery CF, Madden DJ, Schniebolk S. Long-term effects of exercise on psychological functioning in older men and women. J Gerontol 1991;46(6):P352-P361. [PubMed: 1940092]

15. Colcombe S, Kramer AF. Fitness effects on the cognitive function of older adults: a meta-analytic study. Psychol Sci 2003;14(2):125-130. [PubMed: 12661673]

16. Colcombe SJ, Kramer AF, Erickson KI, et al. Cardiovascular fitness, cortical plasticity, and aging. Proc Natl Acad Sci U S A 2004;101(9):3316-3321. [PubMed: 14978288]

17. Dishman RK, Berthoud HR, Booth FW, et al. Neurobiology of exercise. Obesity 2006;14(3):345356. [PubMed: 16648603]

18. Doraiswamy PM, Krishnan KR, Oxman T, et al. Does antidepressant therapy improve cognition in elderly depressed patients? J Gerontol A Biol Sci Med Sci 2003;58(12):M1137-M1144. [PubMed: 14684712]

19. Drane DL, Yuspeh RL, Huthwaite JS, Klingler LK. Demographic characteristics and normative observations for derived-trail making test indices. Neuropsychiatry Neuropsychol Behav Neurol 2002;15(1):39-43. [PubMed: 11877550]

20. Dunn AL, Trivedi MH, Kampert JB, Clark CG, Chambliss HO. Exercise treatment for depression: efficacy and dose response. Am J Prev Med 2005;28(1):1-8. [PubMed: 15626549]

21. Dustman RE, Ruhling RO, Russell EM, et al. Aerobic exercise training and improved neuropsychological function of older individuals. Neurobiol Aging 1984;5(1):35-42. [PubMed: 6738784]

22. Emery CF, Gatz M. Psychological and cognitive effects of an exercise program for communityresiding older adults. Gerontologist 1990;30(2):184-188. [PubMed: 2347498]

23. Emery CF, Schein RL, Hauck ER, MacIntyre NR. Psychological and cognitive outcomes of a randomized trial of exercise among patients with chronic obstructive pulmonary disease. Health Psychol 1998;17(3):232-240. [PubMed: 9619472]

24. Etnier JL, Nowell PM, Landers DM, Sibley BA. A meta-regression to examine the relationship between aerobic fitness and cognitive performance. Brain Res Brain Res Rev 2006;52(1):119-130.

25. Fabre C, Chamari K, Mucci P, Masse-Biron J, Prefaut C. Improvement of cognitive function by mental and/or individualized aerobic training in healthy elderly subjects. Int J Sports Med 2002;23 (6):415-421. [PubMed: 12215960]

26. Golden, CJ.; Freshwater, SM. Stroop Color and Word Test: A Manual for Clinical and Experimental Uses. Wood Dale, IL: Stoelting; 2002. p. 1-68. 
27. Gorenstein C, de Carvalho SC, Artes R, Moreno RA, Marcourakis T. Cognitive performance in depressed patients after chronic use of antidepressants. Psychopharmacology 2006;185(1):84-92. [PubMed: 16485140]

28. Gualtieri CT, Johnson LG, Benedict KB. Neurocognition in depression: patients on and off medication versus healthy comparison subjects. J Neuropsychiatry Clin Neurosci 2006;18(2):217-225. [PubMed: 16720799]

29. Hamilton M. A rating scale for depression. J Neurol Neurosurg Psychiatry 1960;23:56-62. [PubMed: 14399272]

30. Hassmen P, Ceci R, Backman L. Exercise for older women: a training method and its influences on physical and cognitive performance. Eur J Appl Physiol 1992;64(5):460-466.

31. Hawkins HL, Kramer AF, Capaldi D. Aging, exercise, and attention. Psychol Aging 1992;7(4):643653. [PubMed: 1466833]

32. Heaton, RK.; Miller, W.; Taylor, MJ.; Grant, I. Revised Comprehensive Norms for an Expanded Halstead-Reitan Battery: Demographically Adjusted Neuropsychological Norms for African American and Caucasian Adults. San Antonio, TX: Psychological Assessment Resources, Inc.; 2004. p. 1-379.

33. Heyn P, Abreu BC, Ottenbacher KJ. The effects of exercise training on elderly persons with cognitive impairment and dementia: a meta-analysis. Arch Phys Med Rehabil 2004;85(10):1694-1704. [PubMed: 15468033]

34. Hill RD, Storandt M, Malley M. The impact of long-term exercise training on psychological function in older adults. J Gerontol 1993;48(1):P12-P17. [PubMed: 8418145]

35. Hillman CH, Kramer AF, Belopolsky AV, Smith DP. A cross-sectional examination of age and physical activity on performance and event-related brain potentials in a task switching paradigm. Int J Psychophysiol 2006;59(1):30-39. [PubMed: 16413382]

36. Ismail AH, El-Naggar AM. Effect of exercise on cognitive processing in adult men. J Hum Ergol 1981;10:83-91.

37. Khatri P, Blumenthal JA, Babyak MA, et al. Effects of exercise training on cognitive functioning among depressed older men and women. J Aging Phys Act 2001;9(1):43-57.

38. Kramer AF, Colcombe SJ, McAuley E, Scalf PE, Erickson KI. Fitness, aging and neurocognitive function. Neurobiol Aging 2005;26(124):7.

39. Kramer AF, Hahn S, Cohen NJ, et al. Ageing, fitness and neurocognitive function. Nature 1999;400 (6743):418-419. [PubMed: 10440369]

40. Lezak, MD. Neuropsychological Assessment. Vol. 3rd ed.. Oxford: Oxford University Press; 1995. p. 333-601.

41. Madden DJ, Blumenthal JA, Allen PA, Emery CF. Improving aerobic capacity in healthy older adults does not necessarily lead to improved cognitive performance. Psychol Aging 1989;4(3):307-320. [PubMed: 2803624]

42. Mandelli L, Serretti A, Colombo C, et al. Improvement of cognitive functioning in mood disorder patients with depressive symptomatic recovery during treatment: an exploratory analysis. Psychiatry Clin Neurosci 2006;60(5):598-604. [PubMed: 16958944]

43. Moul JL, Goldman B, Warren B. Physical activity and cognitive performance in the older population. J Aging Phys Act 1995;3(2):135-145.

44. Netz Y, Wu MJ, Becker BJ, Tenenbaum G. Physical activity and psychological well-being in advanced age: a meta-analysis of intervention studies. Psychol Aging 2005;20(2):272-284. [PubMed: 16029091]

45. Okumiya K, Matsubayashi K, Wada T, Kimura S, Doi Y, Ozawa T. Effects of exercise on neurobehavioral function in community-dwelling older people more than 75 years of age. $\mathrm{J}$ Am Geriatr Soc 1996;44(5):569-572. [PubMed: 8617907]

46. Paelecke-Habermann Y, Pohl J, Leplow B. Attention and executive functions in remitted major depression patients. J Affect Disord 2005;89(1-3):125-135. [PubMed: 16324752]

47. Palleschi L, Vetta F, De Gennaro E, Idone G. Effect of aerobic training on the cognitive performance of elderly patients with senile dementia of Alzheimer type. Arch Gerontol Geriatr 1996;5:47-50. [PubMed: 18653007] 
48. Palmer A. The effect of an aerobic exercise program on cognitive ability and creativity in senior citizens. Diss Abstr Int B Sci Eng 1995;56(5B):2928.

49. Pereira AC, Huddleston DE, Brickman AM, et al. An in vivo correlate of exercise-induced neurogenesis in the adult dentate gyrus. Proc Natl Acad Sci 2007;104(13):5638-5643. [PubMed: 17374720]

50. Perri S, Templer DI. The effects of an aerobic exercise program on psychological variables in older adults. Int J Aging Hum Dev 1984;20(3):167-172. [PubMed: 6530295]

51. Petruzzello SJ, Landers DM, Hatfield BD, Kubitz KA, Salazar W. A meta-analysis on the anxietyreducing effects of acute and chronic exercise: Outcomes and mechanisms. Sports Med 1991;11(3): 143-182. [PubMed: 1828608]

52. Powell RR. Psychological effects of exercise therapy upon institutionalized geriatric mental patients. J Gerontol 1974;29(2):157-161. [PubMed: 4811950]

53. Rapp MA, Dahlman K, Sano M, Grossman HT, Haroutunian V, Gorman JM. Neuropsychological differences between late-onset and recurrent geriatric major depression. Am J Psychiatry 2005;162 (4):691-698. [PubMed: 15800140]

54. Rikli RE, Edwards DJ. Effects of a three-year exercise program on motor function and cognitive processing speed in older women. Res Q Exerc Sport 1991;62(1):61-67. [PubMed: 2028094]

55. Ruff RM, Niemann H, Allen CC. The Ruff 2 and 7 Selective Attention Test: A neuropsychological application. Percept Mot Skills 1992;75:1311-1319. [PubMed: 1484803]

56. SAS Institute Inc. SAS/STAT 9.1 User's Guide. Cary, NC: SAS Institute Inc; 2004. p. 1-384.

57. Schatzberg AF, Posener JA, DeBattista C, Kalehzan BM, Rothschild AJ, Shear PK. Neuropsychological deficits in psychotic versus nonpsychotic major depression and no mental illness. Am J Psychiatry 2000;157(7):1095-1100. [PubMed: 10873917]

58. Singh NA, Stavrinos TM, Scarbek Y, Galambos G, Liber C, Fiatarone Singh MA. A randomized controlled trial of high versus low intensity weight training versus general practitioner care for clinical depression in older adults. J Gerontol A Biol Sci Med Sci 2005;60(6):768-776. [PubMed: 15983181]

59. Skrinar, GS. Mental Illness. In: Durstine, JL.; Moore, GE., editors. ACSM's Exercise Management for Persons with Chronic Diseases and Disabilities. Champaign, IL: Human Kinetics; 2003. p. 230-232.

60. Stins JF, van Baal GC, Polderman TJ, Verhulst FC, Boomsma DI. Heritability of Stroop and flanker performance in 12-year old children. BMC Neurosci 2004;5(49):1-8. [PubMed: 14720305]

61. Stroop JR. Studies of interference in serial verbal reactions. J Exp Psychol 1935;18:643-662.

62. U.S. Department of Health and Human Services. Physical Activity and Health: A Report for the Surgeon General. Atlanta, GA: U.S. Department of Health and Human Services, Centers for Disease Control and Prevention, National Center for Chronic Disease Prevention and Health Promotion; 1996. p. 1-278.

63. Wechsler, D. Wechsler Adult Intelligence Scale-Revised. New York: Psychological Corporation; 1981. p. 1-156.

64. Wechsler, D. Wechsler Memory Scale-Revised. New York: Psychological Corporation; 1987. p. $1-150$.

65. Whitehurst M. Reaction time unchanged in older women following aerobic training. Percept Mot Skills 1991;72(1):251-256. [PubMed: 2038520]

66. Williams P, Lord SR. Effects of group exercise on cognitive functioning and mood in older women. Australian and New Zealand Journal of Public Health 1997;21(1):45-52. [PubMed: 9141729]

67. Wroolie TE, Williams KE, Keller J, et al. Mood and neuropsychological changes in women with midlife depression treated with escitalopram. J Clin Psychopharmacol 2006;26(4):361-336. [PubMed: 16855452]

68. Zervas Y, Danis A, Klissouras V. Influence of physical exertion on mental performance with reference to training. Percept Mot Skills 1991;72(3):1215-1221. [PubMed: 1961669] 


\section{TABLE 1}

Factor loadings with individual tests.

\begin{tabular}{lccc}
\hline & $\begin{array}{c}\text { Executive } \\
\text { Function }\end{array}$ & $\begin{array}{c}\text { Verbal Fluency/ } \\
\text { Working Memory }\end{array}$ & $\begin{array}{c}\text { Verbal } \\
\text { Memory }\end{array}$ \\
\hline Digit Symbol & 80 & 40 & 19 \\
Ruff Total & 75 & 34 & 16 \\
Stroop Color-Word & 68 & 46 & 19 \\
TMT B-A & -72 & -53 & -23 \\
Digits Backward & 41 & 71 & 24 \\
Digits Forward & 29 & 62 & 11 \\
COWAT & 38 & 54 & 27 \\
Animal Fluency & 41 & 45 & 35 \\
Verbal Paired Associates, hard & 19 & 28 & 71 \\
Verbal Paired Associates, easy & 7 & 9 & 59 \\
Logical Memory & 21 & 23 & 52 \\
& & & \\
\hline
\end{tabular}




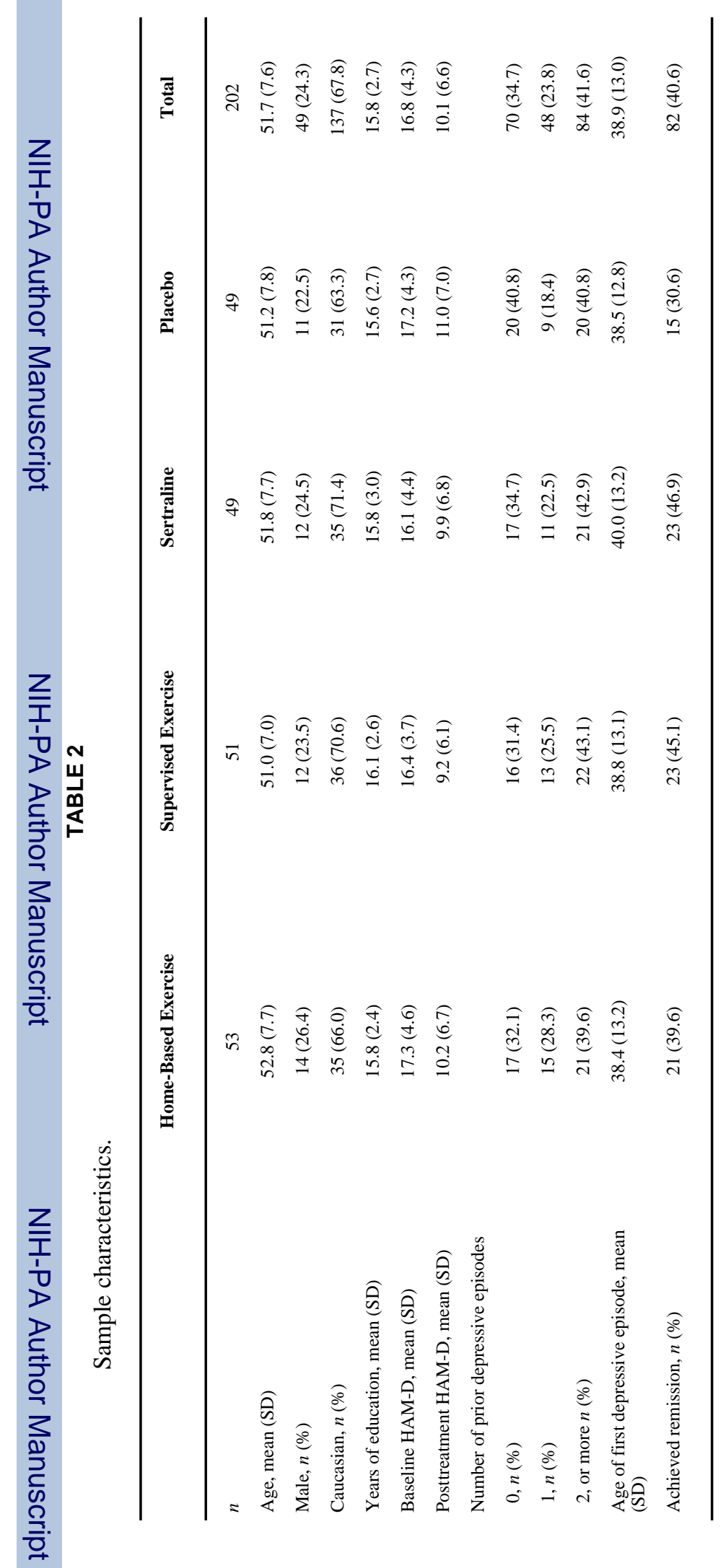


TABLE 3

Mean average treadmill test performance, by group and time.

\begin{tabular}{|c|c|c|c|c|}
\hline & $\begin{array}{c}\text { Supervised } \\
\text { Exercise } \\
(\boldsymbol{n}=\mathbf{5 1})\end{array}$ & $\begin{array}{c}\text { Home-Based } \\
\text { Exercise } \\
(\boldsymbol{n}=\mathbf{5 3})\end{array}$ & $\begin{array}{c}\text { Sertraline } \\
(n=49)\end{array}$ & $\begin{array}{l}\text { Placebo } \\
(n=49)\end{array}$ \\
\hline \multicolumn{5}{|c|}{ Peak $\mathrm{VO}_{2}\left(\mathrm{~mL} \cdot \mathrm{min}^{-1} \cdot \mathrm{kg}^{-1}\right)$} \\
\hline Before treatment & 25.2 & 24.8 & 24.2 & 23.9 \\
\hline After treatment & 26.8 & 25.7 & 23.4 & 22.9 \\
\hline \multicolumn{5}{|c|}{ Treadmill test duration (s) } \\
\hline Before treatment & 620 & 591 & 562 & 575 \\
\hline After treatment & 704 & 634 & 558 & 562 \\
\hline
\end{tabular}




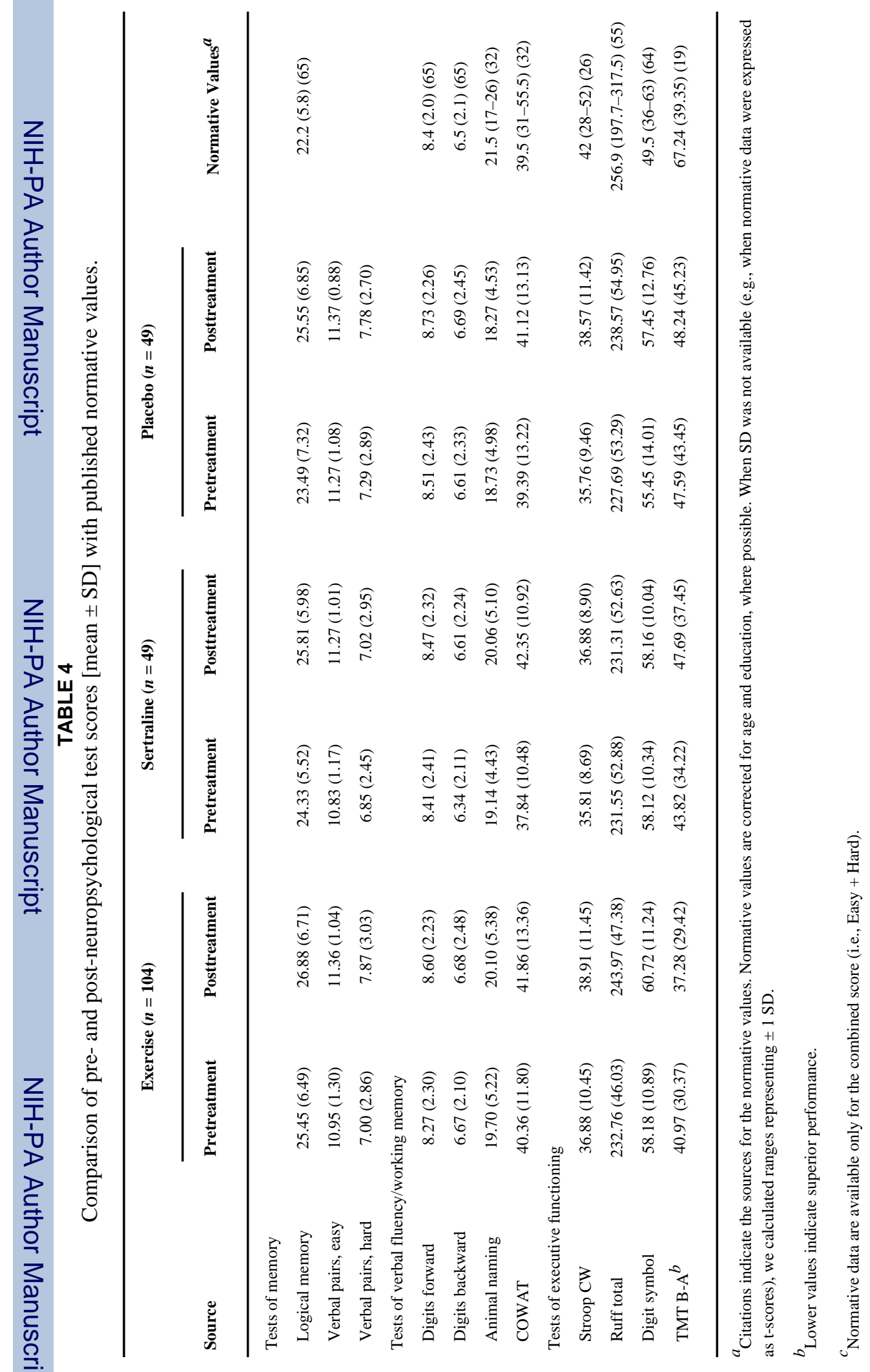

Med Sci Sports Exerc. Author manuscript; available in PMC 2009 September 17. 